\title{
1 Mucosal microbiomes and Fusobacterium genomics in Vietnamese colorectal cancer patients
}

3 Hoang N. H. Tran ${ }^{1 \#}$, Trang Nguyen Hoang Thu ${ }^{1 \#}$, Phu Huu Nguyen ${ }^{2}$, Chi Nguyen $\mathrm{Vo}^{2}$, Khanh Van Doan ${ }^{3}$,

4 Chau Nguyen Ngoc Minh ${ }^{1}$, Ngoc Tuan Nguyen ${ }^{2}$, Van Ngoc Duc Ta ${ }^{2}$, Khuong An Vu ${ }^{2}$, Thanh Danh Hua ${ }^{2}$,

5 To Nguyen Thi Nguyen ${ }^{1}$, Tan Trinh Van ${ }^{1}$, Trung Pham Duc ${ }^{1}$, Ba Lap Duong ${ }^{2}$, Phuc Minh Nguyen ${ }^{2}$, Vinh

6 Chuc Hoang ${ }^{2}$, Duy Thanh Pham ${ }^{1,4}$, Guy E. Thwaites ${ }^{1,4}$, Lindsay J. Hall ${ }^{5,6,7}$, Daniel J. Slade ${ }^{8}$, Stephen

$7 \quad$ Baker $^{9}$, Vinh Hung Tran ${ }^{2}$, Hao Chung The ${ }^{1^{*}}$

8

$9 \quad{ }^{1}$ Oxford University Clinical Research Unit, Ho Chi Minh City, Vietnam

$10 \quad{ }^{2}$ Binh Dan Hospital, Ho Chi Minh City, Vietnam

$11 \quad{ }^{3}$ Department of Oral Biology, Yonsei University College of Dentistry, Seoul, Korea

$12{ }^{4}$ Centre for Tropical Medicine and Global Health, Nuffield Department of Clinical Medicine, University of Oxford,

13 Oxford, United Kingdom

$14 \quad{ }^{5}$ Quadram Institute Biosciences, Norwich Research Park, Norwich, United Kingdom

$15 \quad{ }^{6}$ Norwich Medical School, University of East Anglia, Norwich Research Park, Norwich, United Kingdom

$16{ }^{7}$ Intestinal Microbiome, School of Life Sciences, ZIEL - Institute for Food \& Health, Technical University of

17 Munich, Freising, Germany

$18 \quad{ }^{8}$ Department of Biochemistry, Virginia Tech, Blacksburg, VA 24061, USA

$19{ }^{9}$ Department of Medicine, Cambridge Institute of Therapeutic Immunology and Infectious Diseases (CITIID),

20 University of Cambridge, Cambridge, United Kingdom

21

22 Hoang N.H. Tran and Trang Nguyen Hoang Thu contributed equally to this article.

$23 *$ Corresponding author: Dr. Hao Chung The, Department of Molecular Epidemiology, Oxford University Clinical

24 Research Unit (OUCRU), 764 Vo Van Kiet St., Ward 1, District 5, Ho Chi Minh City, Vietnam.

25 Tel: +84969937143 Email: haoct@ oucru.org 


\section{Abstract}

29 Perturbations in the gut microbiome have been linked to the promotion and prognosis of colorectal cancer

30 (CRC), with the colonic overabundance of Fusobacterium nucleatum shown as the most consistent

31 marker. Despite the increasing health burden inflicted by CRC in low- and middle-income countries like

32 Vietnam, the CRC-specific microbiome in these populations remains underexplored. Here we conducted a

33 study in Vietnam to enrol 43 CRC patients (cases) and 25 patients with non-cancerous colorectal polyps

34 (controls) between December 2018 and January 2020. Our study investigated the mucosal microbiome

35 signature and genomic diversity of Fusobacterium in Vietnamese CRC patients, using a combination of

36 16S rRNA gene profiling, anaerobic microbiology, and whole genome sequencing. We found that several

37 oral bacteria, including F. nucleatum and Leptotrichia, were significantly more abundant in the tumour

38 mucosa, and these two bacteria were also more enriched in tumours of advanced CRC stages (III-IV). We

39 obtained 53 Fusobacterium genomes from the saliva, tumour and non-tumour mucosa of six CRC patients.

40 Isolates from the gut mucosa belonged to diverse $F$. nucleatum subspecies (nucleatum, animalis, vincentii,

41 polymorphum) and a potential new subspecies of $F$. periodonticum. The Fusobacterium population within

42 each individual was distinct and in many cases diverse, with minimal intra-clonal variation. Phylogenetic

43 analyses showed that within each individual, tumour-associated Fusobacterium were clonal to those

44 isolated from non-tumour mucosa, but distantly related to those isolated from saliva. Genes encoding

45 major virulence factors (Fap2 and RadD) showed variability in length and evidence of horizontal gene

46 transfer. Our work provides a framework to understand the genomic diversity of Fusobacterium within

47 the CRC patients, which can be exploited for the development of CRC diagnostic and therapeutic options

48 targeting this oncobacterium.

49

50 Keywords: colorectal cancer microbiome; Fusobacterium; Leptotrichia; Fusobacterium genomic;

51 developing country; Fusobacterium diversity; cancer microbiome; mucosal microbiome 


\section{Introduction}

53 Colorectal cancer (CRC) is the second leading cause of cancer mortality worldwide, contributing to an

54 estimate of 850,000 deaths and $\sim 1.8$ million new cases in 2018 [1,2]. The majority of CRC cases are

55 sporadic (without clear heredity components), with well-established lifestyle risk factors attributed to

56 obesity, alcohol consumption and a diet enriched with red or processed meat [3]. The vast and diverse

57 microbial community inhabiting the colon (termed the gut microbiome) is an integral part of human

58 health, and act as an important interface mediating the interactions between environmental cues, host

59 biology, and CRC [4,5]. Research on CRC gut microbiome has consistently underlined the abundances of

60 certain marker bacteria, among which Fusobacterium nucleatum has been most widely reported and

61 intensively studied [6-10].

62

63 The Gram-negative rod-shaped F. nucleatum is a common anaerobic member of the human oral

64 microbiome, and it is currently composed of four subspecies (nucleatum, vincentii, animalis, and

65 polymorphum) [11]. Mechanistic studies have demonstrated that $F$. nucleatum possesses several virulence

66 factors, most notably FadA and Fap2, which enable the bacteria to potentiate colonic tumourigenesis. The

67 adhesin FadA binds to E-cadherin in CRC cells and activates the $\beta$-catenin-dependent oncogenic

68 pathways [12], while the lectin Fap2 further facilitates $F$. nucleatum invasion into CRC cells by

69 specifically binding to the tumour-enriched carbohydrate Gal-GalNAc [13]. Such interaction triggers the

70 secretions of the pro-inflammatory (IL-8) and pro-metastatic (CXCL-1) cytokines, creating a tumour

71 environment conditioned for accelerated growth and migratory tendency [14]. Recent studies have further

72 highlighted that the bacteria could induce DNA damage in oral and colorectal cancerous cells $[15,16]$.

73 Additionally, F. nucleatum lipopolysaccharide was shown to induce resistance to chemotherapy via

74 activation of the autophagy machinery in CRC cells, thus complicating effective CRC treatment [17]. As

75 a result, enrichment of $F$. nucleatum in CRC microbiomes has been associated with more severe

76 prognosis and poorer overall survival, particularly in a subset of patients with mesenchymal tumours [18-

77 20]. Preclinical research demonstrated that $F$. nucleatum elimination by antibiotics reduced colorectal 
78 tumour proliferation in mice [21]. These evidences strongly support for the utilization of $F$. nucleatum as

79 a target for CRC diagnosis and therapy, but current translational potential is hampered by the lack of

80 insights into F. nucleatum diversity and its genomic characteristics in CRC patients.

81

82 The majority of microbiome studies, on either healthy or CRC cohorts, were conducted in high-income

83 countries, and such data are sparse regarding populations in developing settings, where host factors, diet

84 and lifestyle could greatly influence the gut microbiome composition and function. Vietnam has an

85 increasing ageing population adopting a more 'Westernized' diet and sedentary lifestyle [22], where CRC

86 incidence is predicted to climb and rank as among the top three cancers by 2025 [23]. Therefore, CRC

87 microbiome studies in Vietnam are necessary to establish the basis for the implementation of

88 microbiome-oriented strategies for CRC prevention, diagnosis, prognosis and therapy. We set out to

89 investigate the microbiome signatures of Vietnamese CRC patients, by applying 16S-rRNA gene

90 profiling on the saliva and gut mucosa collected from patients with CRC and non-cancerous colorectal

91 polyps. Additionally, different from prior studies, we used anaerobic culturing and whole genome

92 sequencing (WGS) to study the genomic diversity of Fusobacterium colonizing these CRC patients,

93 allowing an in-depth and high-resolution examination of these bacterial populations. 


\section{Results}

\section{Gut mucosal, but not salivary, microbiomes differ significantly between CRC and controls}

96 We enrolled 43 CRC patients (cases) and 25 patients with colorectal polyps (controls) between December

972018 and January 2020. 16S rRNA microbiome profiling was performed for all the saliva and gut mucosa

98 samples collected from the participants, including tissues originating from the diseased (CRC tumour or

99 polyps) and the adjacent normal sites. To limit the scope of this study, we selected participants with

100 tumours/polyps detected in the distal colon or rectum. The patients' demographic and clinical data were

101 summarized in Table 1, which showed that there were no significant differences between the two groups.

102 All polyps showed not more than low-grade dysplasia (i.e. non-cancerous), demonstrating the validity of

103 our control group. Microbiome profiling identified 865 filtered amplicon sequence variants (ASVs - a

104 marker for distinct taxonomic classification) among 66 saliva samples, with a median library size of

10536,250 paired-end reads [IQR: 31,827 - 50,317]. Due to their lower microbial biomass, the library size of

106 gut mucosal microbiomes was smaller (median: 17,711 [IQR: 9,037 - 30,135]), with 1,073 filtered ASVs

107 detected across 129 mucosa samples (seven removed).

109 Ordination by principal coordinate analysis (PCoA), based on phylogenetic-assisted isometric log-ratio

110 (PhIRL) transformed value, showed that the salivary microbiomes of CRC and controls completely

111 overlapped (Figure 1A). Only active smoking within the last two years, but not CRC status, was

112 significantly associated with the salivary microbiome structure (RDA, p-value $=0.033)$. Likewise, only

113 two ASVs belonging to the genera Leptotrichia and Solobacterium were consistently identified as

114 significantly more abundant in the CRC's salivary microbiome. These point to the high structural

115 similarity in the salivary microbiome between the two groups. By contrast, the gut mucosal microbiomes

116 differ significantly based on CRC status (Figure 1B). CRC and diabetes significantly contributed to the

117 variance in the gut microbiome (RDA, p-value < 0.05). Gut mucosa collected within a participant (tumour

118 and non-tumour for CRC, biopsy and polyp for control) shared more similarity in their microbiomes than

119 those of the same sample type between participants (Figure 1C), resembling findings from previous 
120 research [8]. We also conducted these analyses using the weighted Unifrac and Bray-Curtis distances,

121 which produced similar interpretations. Additionally, we performed unsupervised clustering on gut

122 mucosa microbiomes, which showed the presence of two robust community state types (CSTs) supported

123 by an out-of-bag error rate of $10.8 \%$ in a random forest classification. This algorithm also identified that

124 several 'balances’ (Proteobacteria/Actinobacteria, other bacteria/Lachnospiraceae) contributed

125 significantly in separating the two CSTs (Figure S1). CST1 was generally more enriched in

126 Gammaproteobacteria (mostly Escherichia) while CST2 had higher abundance of Actinobacteria (mainly

127 Collinsella) and Lachnospiraceae (Figure 1D). The two CSTs were similar in library size (p-value $=0.15$,

128 t-test), but different in CRC status (p-value=0.002, Fisher-exact test), with the majority of control samples

$129(72 \%)$ belonging to CST1. No other tested covariates were associated with CST grouping. Samples from

130 the same patients mostly shared the same CST membership $(90.3 \%, \mathrm{n}=56 / 62$ patients with paired

131 microbiomes), and CRC samples were distributed in both CSTs with different proportion $(\mathrm{CST} 1=36$,

132 CST2 = 50). These findings suggest that while CRC status mainly explained the dissimilarity observed in

133 the gut mucosal microbiomes, their overall configurations were determined by the dominant presence of

134 Gammaproteobacteria (Escherichia), possibly driven by an unknown or stochastic factor.

\section{Enrichment of oral bacteria in the tumour gut mucosa}

137 We applied differential abundance analysis to rigorously detect bacteria enriched in the CRC tumours, by 138 comparing results from different approaches, including ANCOMBC, DESeq2 and corncob (see Methods)

139 [24-26]. Our analyses revealed that ASVs classified as bacteria of putative oral origin (Gemella,

140 Peptostreptococcus, F. nucleatum, Leptotrichia, Selenomonas sputigena, and Campylobacter rectus)

141 were overabundant in the tumour mucosa, compared to control biopsies (Figure 2B). This finding

142 corroborates the observation that these oral bacteria had a higher relative abundance in CRC gut

143 microbiomes of both CSTs (Figure 1D). Within the CRC patients, tumours also showed an elevated

144 presence of the aforementioned oral bacteria (alongside Hungatella, Lachnoclostridium, and Osillibacter)

145 when compared to adjacent non-tumour mucosa, albeit with less pronounced fold change (Figure 2A). 
146 These increases were coupled with the reduction in abundances of commensal anaerobes in the tumour

147 mucosa, such as Blautia, Parabacteroides, Dorea, and Collinsella. When comparing between different

148 cancer stages, the increased abundance of one taxon (Leptotrichia, ASV-13) was consistently associated

149 with tumours of advanced stages (III-IV), compared to stage II (Figure 2C). Results from DESeq2 alone

150 additionally showed that $F$. nucleatum was also enriched in advanced CRC stages (adjusted p-value

$151<0.05)$. ASVs confidently assigned as F. nucleatum $(\mathrm{n}=14)$ and Leptotrichia spp. $(\mathrm{n}=16)$ were present

152 with at least $0.1 \%$ relative abundance in $\sim 54 \%$ and $40 \%$ of tumour microbiomes, respectively. On the

153 other hand, tumour samples with low abundances of oral bacteria $(<1 \%)$ were dominated by other taxa,

154 including Escherichia/Klebsiella $(\mathrm{n}=4)$, Megamonas $(\mathrm{n}=4)$, Fusobacterium mortiferum $(\mathrm{n}=2)$, and

155 Helicobacter $(\mathrm{n}=1)$. We performed similar analysis within the control group and showed that only one

156 ASV (Faecalibacterium) was consistently depleted in polyps compared to paired biopsies. However,

157 when compared to CRC samples, F. mortiferum, Tyzzerella, and Sutterella were significantly enriched in

158 the control gut microbiomes (Figure 2B).

To investigate bacterial co-occurrence and their potential interactions, we next used CCLasso and

161 SpiecEasi to construct a correlation network of gut microbiomes from CRC patients ( $\mathrm{n}=86$ ) (Figure 3)

162 [27,28]. Two oral bacteria clusters emerged from this network, one consisting of several Streptococcus

163 and Veillonella taxa, and another composed mostly of aforementioned tumour-associated ASVs

164 (Leptotrichia, Selenomonas, F. nucleatum, Streptococcus, Granulicatella, Gemella, Peptostreptococcus,

165 and Parvimonas). The latter cluster exhibited positive correlation with E. coli, and antagonism toward

166 Blautia, a member of the gut anaerobic commensal network. Besides, other tumour-associated ASVs such

167 as Hungatella, Lachnoclostridium, and C. rectus were clustered alongside Negativibacillus and

168 Eggerthella, which showed strong negative correlations with anaerobic gut commensals Dorea,

169 Bacteroides, and Faecalibacterium. These findings highlight the potential competition between tumour-

170 associated taxa and common gut commensal anaerobes (Lachnospiraceae, Oscillospirales). Other

171 Fusobacterium species, F. mortiferum and F. varium were not linked to the oral clusters, showing that 
172 they were mainly gut inhabitants. Comparison with the network constructed from salivary microbiomes

173 revealed that the same tumour-associated ASVs (F. nucleatum, Gemella, Selenomonas) formed similar

174 clusters as observed in the CRC gut microbiomes (Figure S2). This indicates that tumour-associated

175 ASVs could have oral origin in this examined cohort, and they likely co-exist in the polymicrobial

176 biofilms (similar to those present in the oral cavity) upon gut colonization. Notably, the tumour-

177 associated Leptotrichia (ASV-13) had very low abundance in the salivary microbiome (mean: 0.037\%,

178 prevalence: 26\%), and it differs from the dominant Leptotrichia detected in saliva (ASV-19) in 17

179 nucleotides (pairwise similarity: 93.3\%).

\section{Diverse Fusobacterium colonizes CRC patients}

182 Since $F$. nucleatum was more enriched in the tumour microbiomes and previously demonstrated to

183 promote tumourigenesis, we next studied the population structure of Fusobacterium recovered from CRC

184 patients. Six patients with a Fusobacterium relative abundance at the tumour site exceeding 10\% (except

185 for patient 18) and covered different cancer stages were selected for Fusobacterium isolation. In total, we

186 isolated 56 presumptive Fusobacterium organisms, as identified by the matrix-assisted laser

187 desorption/ionization time of flight mass spectrometer (MALDI-TOF), from the oral, nontumour and

188 tumour samples of these patients (Table 2). Whole genome short-read sequencing was performed on these

189 isolates, and three were determined contaminated and removed from analyses. Fifty-three recovered

190 genomes belong to $F$. nucleatum $(\mathrm{n}=38)$ and $F$. periodonticum $(\mathrm{n}=15)$ species complexes, of which

191 phylogenetic reconstruction was performed separately. Core-genome phylogeny of $F$. nucleatum showed

192 that tumour-associated isolates were detected in all four subspecies (animalis, vincentii, nucleatum,

193 polymorphum) (Figure $4 \mathrm{~A})$. In the $F$. periodonticum phylogeny, tumour-associated isolates $(\mathrm{n}=8$, isolated

194 from P18, P40) formed a distinct cluster that is phylogenetically separated from the available references

195 (Figure 4B). These isolates all showed $\sim 91 \%$ average nucleotide identity (ANI) to the closest $F$.

196 periodonticum references, suggesting that they constitute a novel subspecies of this species complex,

197 denoted herein as novel F. periodonticum (novelFperi). Likewise, two gut isolates (H16-13, H16-14) 
shared $93 \%$ ANI to the closest $F$. nucleatum references and were phylogenetically distant from the remaining $F$. nucleatum isolates, potentially indicative of a novel $F$. nucleatum subspecies. Across the two phylogenies, we identified 14 phylogenetic clusters (PCs; $2-6$ isolates each) and 12 singletons

201 originating from this study's collection, which were collectively named as PCs herein.

204 Fusobacterium species/subspecies were detected in each patient's saliva, sometimes with more than one

205 PCs of the same subspecies (P18, P46) (Table 2). Likewise, we observed similar diversity in gut-

206 associated isolates, with more than one PCs detected in three patients (P10, P16, P18). Most patients did

207 not share the same Fusobacterium subspecies recovered from both oral- and gut-associated isolates,

208 except for P16 (polymorphum). However, phylogenetic evidence confirmed that the two niches harboured

209 distinct populations, which were 16,955 SNPs apart (Figure 4A). Particularly, oral Fusobacterium

210 isolates from P18 (n=9) belonged to six different PCs (mostly F. periodonticum and $F$. hwasookii), while

$2116 / 7$ gut isolates were of a single novelFperi clone. By contrast, Fusobacterium from tumour and

212 nontumour sites were frequently clustered in the same PC ( $\mathrm{n}=4$; in P10, P16, P18 and P40), indicating

213 that the same bacterial clones have colonized and spread beyond the tumour microenvironment. We used

214 the mapping approach to confidently inspect the intraclonal variations within these PCs, and showed that

215 they shared minimal genetic differences in the core genome (1 - 2 SNVs). These values fall in range with

216 the variation observed in five other gut PCs (with either tumour or nontumour isolates; 0 - 5 SNVs) and

217 five other oral PCs (1 - 10 SNVs).

219 Variation in Fusobacterium virulence gene content

220 We next sought to examine the presence of several Fusobacterium virulence factors, of which

221 pathogenicity has been proven in experimental studies, including genes encoding adhesin $(f a d A, c b p F)$,

222 lectin (fap2), and bacterial co-aggregation factor ( $\operatorname{radD})[12,13,29,30]$. RadD is an autotransporter

223 facilitating Fusobacterium's interspecies interaction in polymicrobial biofilms [30], while CbpF inhibits 
$224 \mathrm{CD}^{+} \mathrm{T}$-cell response through CEACAM1 binding and activation [31]. Genomic screening showed that

225 fap2 was present and intact in the majority of genomes from both species (49/53), with disruptive

226 mutations occurring in some isolates, such as the tumour-associated F. nucleatum animalis in P46 (Figure

227 4A). We also detected $\mathrm{fadA}$ in all isolates (except S18-65), with all $F$. periodonticum variants one amino

228 acid shorter (codon A22) than the canonical FadA found in F. nucleatum (129 aa). The other elements

229 showed variable presence among the examined genomes. For example, $c b p F$ was present in all $F$.

230 nucleatum nucleatum, F. nucleatum vincentii, and novelFperi, while radD was co-localised with

231 fadA2/radA (a 122 aa fadA homolog) in 28 isolates. Another fadA homolog (fadA3) with unknown

232 function was prevalent in both two Fusobacterium species. Phylogenies of FadA and CbpF showed that

233 the two tree topologies were largely in agreement with those inferred from the core genomes, suggesting

234 the absence of horizontal gene transfer (Figure S3). By contrast, the clustering pattern observed in the

235 Fap2 phylogeny was concordant to subspecies classification for $F$. nucleatum nucleatum, $F$. nucleatum

236 vincentii, and $F$. periodonticum, but was admixed for $F$. nucleatum polymorphum, $F$. hwasookii and $F$.

237 nucleatum animalis (Figure S4A). fap2 encodes a very large protein of variable length (median of 3938 aa

238 [range: 3436 - 4669]), and the protein length showed some correlation with its phylogenetic clustering,

239 with variants $>4200$ aa $(\mathrm{n}=6)$ all belonging to a monophyly composed of $F$. hwasookii and $F$. nucleatum

240 polymorphum. Similarly, the RadD phylogeny did not concur with those inferred from the core genomes,

241 and its length variation (median 3526 aa [range: 3461 - 3602]) also showed association with the tree

242 topology (Figure S4B). radD was $\sim 800$ bp downstream of $f a d A 2$, which is flanked by an IS150

243 transposase on the $F$. nucleatum 23726 reference genome. This could explain the mobilization mechanism

244 of radD-fadA2 across the Fusobacterium phylogeny. These data indicate that the autotransporter

245 encoding genes fap 2 and $r a d D$ may have undergone frequent horizontal gene transfer or recombination in

246 the F. nucleatum species complex. 


\section{Discussion}

248 Our study revealed the composition of microbiome perturbations at the tumour mucosa of Vietnamese

249 patients with CRC and non-cancerous colorectal polyps. Tumour-enriched taxa include mostly bacteria of

250 putative oral origin, such as F.nucleatum, Leptotrichia, Gemella, C. rectus, and Selenomonas, which

251 agrees with findings from previous studies profiling either gut mucosal or faecal microbiomes in different

252 CRC populations $[8,9,32]$. This suggests that the proliferation of oral bacteria at the gut mucosa could be

253 a universal signature of CRC microbiomes. Several of these oral taxa shared identical ASVs between the

254 oral and gut niches, pointing to the oral origin of tumour-associated taxa. Our analysis found that these

255 bacteria also display a co-occurrence pattern, indicating that they likely co-exist in a biofilm-like

256 aggregate upon colonization at the gut mucosa. Indeed, previous research has confirmed the frequent

257 presence of polymicrobial biofilms composed of oral taxa (F.nucleatum, Peptostreptococcus, Gemella)

258 in colorectal tumour tissues [33]. Among the oral bacteria, F. nucleatum stands out for its ability to form

259 "bridging" interactions with other bacteria via the presence of several adhesins [11]. F. nucleatum was

260 recently reported to secrete FadA with amyloid properties, which confers acid tolerance and provides a

261 scaffold for biofilm formation [34]. In addition, our analyses pointed to the significant presence of

262 Leptotrichia in tumour mucosa, especially in advanced tumours. This association, however, has only been

263 noted in few studies [32,35]. This may be due to the differences in sampling location, as tumours excised

264 from the distal colon (as performed for all cases in our study) were reported to harbour a higher

265 abundance of Leptotrichia, compared to those originating from the proximal colon [35]. The

266 overabundance of Leptotrichia in the salivary microbiome has been implicated in patients with malignant

267 oral leukoplakia and pancreatic cancer [36,37], as well as with CRC as shown in this study. Leptotrichia

268 belongs to the same order as Fusobacterium (Fusobacteriales) and could carry virulence factors similar to

269 those found in the latter genus. It is noteworthy that the predominant tumour-associated Leptotrichia

270 taxon (ASV-13) could be detected from different CRC patients, but was in very low abundance in these

271 patients' salivary microbiomes. This suggests that a distinct Leptotrichia species/genotype was associated

272 with CRC, which warrants more in-depth investigations. 
274 Asides from oral taxa, Hungatella overabundance was the most significant signature of CRC microbiome

275 in our dataset. This falls in line with results from a recent metagenomic meta-analysis, showing that

276 Hungatella hathewayi's specific choline trimethylamine-lyase gene (cutC) was significantly enriched in

277 the faecal microbiomes of CRC patients [10]. Moreover, colonic H. hathewayi could induce

278 hypermethylation in prominent tumour suppressor genes, thus silencing their functions and promoting

279 intestinal epithelial cell proliferation [38]. Combining quantitative detection of microbial markers $(H$.

280 hathewayi, F. nucleatum, Lachnoclostridium, and Bacteroides clarus) with faecal immunochemical test

281 greatly increased sensitivity (reaching 94\%) for diagnosing CRC in a Chinese population [39].

282 Consistent with this finding, our analyses suggested that Hungatella and Lachnoclostridium were

283 overabundant and co-occurring in the tumour mucosa of the Vietnamese cohort, which supports the

284 feasibility of applying such microbial detection test for non-invasive CRC screening in our setting. On the

285 other hand, we found that $F$. mortiferum was the most significantly enriched taxon in the polyp control

286 group. F. mortiferum was known as a hallmark for dysbiosis in infectious diarrhoea [40], and recent

287 studies have also reported the abundance of $F$. mortiferum in patients with colorectal polyps [41,42].

288 Furthermore, this species was shown to be present in the gut microbiomes of $\sim 60 \%$ of a cohort in

289 Southern China, albeit in very low abundance ( 0.5\%) [43]. Unlike other Fusobacterium species, $F$.

290 mortiferum was devoid of distinctive virulence factors such as adhesins FadA and Fap2 [44], but could

291 utilize a wide range of sugars for growth independent of amino acid metabolism [45]. The association

292 between F. mortiferum and colorectal polyps will need to be further addressed in future studies.

294 Despite the increasing importance of $F$. nucleatum in the pathogenesis of CRC and other invasive

295 diseases [11], genomic characterization of these bacteria from patient populations is currently limited due

296 to technical difficulties in Fusobacterium isolation. Here, we applied targeted culturomics approach,

297 which combines anaerobic culturing, high-throughput identification by MALDI-TOF and WGS, to study

298 the Fusobacterium population in high resolution and help uncover novel bacteria [46]. Indeed, we 
discovered novel subspecies of both $F$. nucleatum and $F$. periodonticum from culturing the gut mucosa, showing that the microbiomes in non-Western settings offer untapped diversity. Using metagenomic assemblies from Chinese faecal microbiomes, Yeoh and colleagues have proposed several new Fusobacterium species (based on 95\% ANI cutoff) [44]. However, our WGS approach provided more accurate and complete realization of the bacterial genomes, which contributes to the global representation of Fusobacterium diversity (with 26 non-duplicate assemblies added). Furthermore, our approach allows for delineation of bacteria from tumour and non-tumour sites, which is inaccessible by faecal metagenomes. The populations of Fusobacterium colonizing the oral cavity and gut mucosa were heterogeneous within each individual, even at the subspecies level, which mirrors the diversity observed previously for gut commensals such as Bifidobacterium species [47]. Though this study did not provide evidence of genetic relatedness between oral and gut Fusobacterium isolates, this likely point to the high diversity of Fusobacterium in the oral niche [48]. Besides, Fusobacterium is abundant in subgingival

311 dental biofilms, which our salivary sampling did not fully capture. Previous research deploying WGS has

312 demonstrated that oral and tumour-originated $F$. nucleatum shared little genetic divergence, supporting

313 the notion that oncogenic Fusobacterium arise from the patient's oral microbiome [49]. Chronic

314 infections with Helicobacter pylori at the stomach, which increases the risk of gastric cancer, usually

315 result in extensive clonal propagations detected by WGS within each patient, though isolates were

316 collected in a single timepoint [50]. This prolonged colonization scenario contrasts with our observations

317 in CRC, in which multiple Fusobacterium clones (with minimal intraclonal variation) were present at

318 each patient's gut mucosa. Given that CRC could take years to develop, we speculate that the

319 Fusobacterium population at the tumour site fluctuates in response to the frequent seedings from the

320 highly diverse oral source. Longitudinal study design is needed to address this hypothesis, and to further

321 assess how Fusobacterium adapts to the gastrointestinal pressure.

323 The two well-described major virulence genes ( $f a d A$ and fap2) were identified in the majority of

324 Fusobacterium genomes, regardless of niche. This concurs with previous research reporting the high 
325 prevalence of $f a d A$ and fap2 in F. nucleatum and F. periodonticum metagenomic assemblies from a

326 cohort in China [44]. These suggest that Fusobacterium with high virulence potential are prevalent in the

327 human population, and the genetic presence of fadA and fap 2 is not suitable for predicting the risk of

328 Fusobacterium-related CRC. All gut-derived novelFperi isolates harboured the examined virulence genes

$329(f a d A, f a p 2, \operatorname{radD}$, and $c b p F)$, which was more similar to $F$. nucleatum compared to $F$. periodionticum.

330 Moreover, fap2 and radD showed variation in gene length and evidence of horizontal gene transfer,

331 underlying the significance of dynamic evolutionary processes in shaping Fusobacterium's virulence

332 landscape. Since Fap2 orchestrates F. nucleatum invasion into CRC tumour cells via specific binding to

333 Gal-GalNAc, this ligand-receptor interaction was recently proposed as a target for clinical intervention in

334 Fusobacterium-enriched CRC [51]. Interestingly, our genetic analysis predicted that fap2 was either

335 missing or truncated in some gut-associated Fusobacterium isolates, which may indicate the complex

336 lifestyle of Fusobacterium once colonizing the gut environment.

338 Some limitations were notable in our study design. Due to ethical concerns, patients with colorectal

339 polyps were selected as the control group, instead of healthy age-matched individuals. Our interpretations

340 do not extend to cancer in the proximal colon, though previous reports have noted that proximal CRC

341 tumours had a higher Fusobacterium abundance [52]. The sample size of cultured Fusobacterium isolates

342 was moderate and did not include longitudinal sampling, so it was not possible to investigate the bacterial

343 evolution in longer timeframe. Notwithstanding these shortcomings, our study reconfirmed the prominent

344 role of oral anaerobic conglomerates in CRC microbiome in an understudied Asian population, and

345 provided new insights into the genomic diversity of the oncobacterium Fusobacterium. The observed

346 diversity in this organism should be taken into account when designing future diagnostic or therapeutic

347 tools that target Fusobacterium. 


\section{Material and Methods}

\section{Study design and sample collection}

350 This prospective case-control study enrolled adult Vietnamese patients ( $\geq 18$ years old) admitted at Binh

351 Dan Hospital, a large surgical hospital in Ho Chi Minh City Vietnam, from December 2018 to January

352 2020. The study received ethical approval from the Ethics Committee of Binh Dan Hospital. Written

353 informed consent was obtained from all study participants. Cases were defined as patients diagnosed with

354 left-sided colorectal cancer (distal colon and rectum) of stage II onward, who received colectomy

355 treatment and underwent non-antibiotic pre-operative bowel preparation. Controls were patients

356 diagnosed with colorectal polyps (single/scattered non-cancerous polyps at distal colon or rectum), who

357 received polypectomy at the hospital. For both cases and controls, patients were excluded if they (1) had

358 received antimicrobial treatments within two weeks prior to enrolment, (2) had additional gastrointestinal

359 infections or obstructions, or (3) were immunocompromised. Additionally, the study excluded patients

360 who had received chemo- and/or radio-therapy within four weeks prior to enrolment (for cases) and those

361 were diagnosed with familial adenomatous polyposis (controls).

363 Demographic and clinical information were collected from study participants at recruitment. The

364 calculated body mass index (BMI) was categorized based on WHO recommendation for Asian

365 populations [53]. Cancer stage classification was based on the TNM Staging system [54]. A saliva sample

$366(\sim 3 \mathrm{~mL})$ was collected pre-operation from each study participant (by spitting into a sterile container). For

367 cases, the mucosa epithelia at the tumour and adjacent non-tumour (2-10 $\mathrm{cm}$ away from the tumour) sites

368 were collected aseptically from the excised colon. For controls, we collected colorectal polyps and 2-3

369 biopsies of non-polyp mucosal epithelium $(\sim 50 \mathrm{mg})$ during colonoscopy. All clinical samples were stored

370 on ice and transported back to the OUCRU laboratory within 4 hours, then were stored in $-80^{\circ} \mathrm{C}$ until

371 further experiments.

372

373 16S rRNA gene sequencing 
374 We selected 43 cases and 25 controls for microbiome profiling. Total DNA was extracted from the gut

375 mucosa samples $(n=136)$ using the FastDNA spin kit for soil (MP Biomedicals, USA), with bead-beating

376 step on Precellys 24 homogenizer (Bertin Instruments, France). DNA from the saliva samples ( $\mathrm{n}=67$, one

377 missing) was extracted using the ReliaPrep Blood gDNA Miniprep (Promega, USA). For microbiome

378 profiling, all samples underwent primary PCR amplification (30 cycles) using the conventional V4

379 primers (515F-806R) and KAPA HiFi Hot Start DNA polymerase (KAPA Biosystems, USA), and

380 secondary PCR was performed to add dual-indexes (IDT, USA) to each sample, following procedures

381 optimized in a published protocol [55,56]. Additionally, we applied the same procedures to a positive

382 control (eight species Zymo mock community, Zymo Research, USA) and six negative controls (two for

383 each DNA extraction kits, and two no-template PCR amplifications), in order to respectively evaluate the

384 experiment's efficacy and detect contamination from reagents and kits (kitome) [57]. 16S rRNA

385 sequencing was performed for all samples on one run of the Illumina MiSeq platform, to generate $250 \mathrm{bp}$

386 paired-end reads.

\section{Microbiome data analysis}

389 All data analyses were conducted in R (v4.1.1) and Rstudio using multiple packages, including 'dada2', 'phyloseq', 'DESeq2', ‘ANCOMBC', 'corncob', ‘philr', 'ggplot2', ‘vegan', ‘SpiecEasi’ and others [2426,28,58-61]. Generated sequence reads were analysed under the amplicon sequence variant framework

392 (ASV) using DADA2, in which statistically denoised forward and reverse read pairs were merged to 393 create error-corrected ASVs with single-nucleotide resolution [62,63]. We retained ASVs with length 394 ranging from 249 to $256 \mathrm{bp}$, which matches the desired length of the amplified V4 region, and chimeric 395 sequences were detected and removed independently for each sample. Taxonomic assignment (up to the 396 species level) was performed using the RDP Naïve Bayesian Classifier implemented in 'dada2' package, 397 on the SILVA v138 train dataset [64]. Further filtering removed ASVs matching the following criteria (1) 398 classified as 'Mitochondria' or 'Archaea', (2) unclassified at Kingdom or Phylum level, (3) identified as 399 kitome or contamination from mock community (except Escherichia and Enterococcus ASVs), or (4) 
identified as low abundant singletons (abundance $\leq 10$ counts and present in only one sample). This

resulted in 2,461 ASVs detected across 203 samples (68 participants), totalling 5,250,754 sequences.

Saliva and gut mucosal microbiomes were then analysed separately. For saliva microbiomes, we removed singleton ASVs with abundances < 79 sequences and one sample with low sequencing depth (837 sequences). The filtered ASVs ( $\mathrm{n}=865)$ were aligned using PASTA [65], and a maximum likelihood phylogeny was constructed under the GTR+G model using IQ-Tree (with 1,000 rapid bootstrap) [66]. The

407 resulting phylogeny was used to transform the ASV count matrix into isometric log-ratio (ILR) 'balances'

408 (weighted log-ratio between two ASVs), using the "philr" package (with zero counts imputed using the

409 “cmultRepl" function) [60,67]. This transformation allowed statistical analyses to be performed

410 accurately in the Euclidean space [68]. Ordination was performed using principle coordinate analysis

411 (PCoA) on a calculated Euclidean distance matrix, implemented in package 'phyloseq'. To identify

412 covariates which explain the salivary microbiome structures, we performed redundancy analysis on the

413 'balance' value matrix of 62 samples with complete metadata (case-control grouping, age, sex, BMI,

414 presence of oral diseases, and status of active smoking in the last two years). We repeat the same

415 analytical procedures on the gut mucosal microbiome data. Low-abundance singleton ASVs $(<44$

416 sequences) and seven samples with low sequencing depth $(<1,300$ sequences each) were removed,

417 retaining 1,073 ASVs across 129 samples for downstream analyses. We tested the association between

418 covariates and the gut mucosal microbiome structures using redundancy analysis, performed on the ILR-

419 transformed 'balance' values of 120 samples with complete metadata (sample type, age, sex, BMI, history

420 of diarrhea in seven days, diabetes, high blood pressure, active consumption of alcohol in the last two

421 years, and anatomical location of samples). The ILR-transformed values were used to calculate the

422 differences among the samples (beta-diversity), within and between participants. In addition, the gut

423 mucosal microbiomes $(n=129)$ were clustered into community state types (CSTs) using the partition

424 around medoid (pam) algorithm on the calculated ILR-transformed distance matrix, with the optimal

425 number of CSTs $(\mathrm{k}=2)$ determined by gap statistic and average silhouette width (asw) [69]. The random 
426 forest classification algorithm (10,000 trees) was then used to evaluate this clustering performance (in

427 term of error rate) and to identify 'balances' differentiating the two CSTs, using the 'rfsrc' function

428 implemented in package 'randomforestSRC' [70].

\section{Evaluating differential abundances}

431 In order to detect ASVs that showed significantly differential abundance between two examined groups,

432 we utilized the compositional data analysis approach implemented in ANCOMBC [24]. In addition, the

433 same comparisons were performed using DESeq2 and corncob to check for consistent results, as

434 recommended in recent benchmark studies [71,72]. The comparisons include salivary microbiomes in

435 cases $(n=43)$ and controls $(n=23)$; paired tumours $(n=43)$ against adjacent non-tumours $(n=43)$; paired

436 polyps $(n=16)$ against non-polyp biopsies $(n=16)$; tumours $(n=43)$ against non-polyp biopsies $(n=24)$;

437 tumours of cancer stage III-IV $(n=24)$ against stage II $(n=18)$. For paired comparison within cases and

438 controls, the model design was set to " Patient + sample_type" to increase statistical power [73]. Multiple

439 hypothesis testing was corrected using Holm or Benjamini-Hochberg method, setting false discovery rate

440 as 0.05. ANCOMBC and corncob approaches were carried out using default parameters. For DESeq2,

441 count data were normalized using either the native negative binomial distribution (saliva, tumours vs.

442 biopsies) or the zero-inflated negative binomial (ZINB) distribution implemented in the package

443 'zinbwave' (paired samples in cases and controls, between cancer stages) [74]. Library size corrections

444 were estimated using DESeq2's 'poscounts' method. All comparisons were performed using likelihood

445 ratio test, and ASVs with adjusted p-value < 0.05 (and base mean $>20$ for DESeq2) were considered

446 significant hits. To minimize the number of false positives, ASVs which showed significant hits in at least

447 two tested methods were considered differentially abundant and included in final interpretation. We

448 manually compared the ASV sequences of interest to the expanded Human Oral Microbiome Database

449 (HOMD; www.homd.org/), and species identification was assigned if the ASV showed >99\% nucleotide

450 similarity to that in the database. 


\section{Correlation network}

453 We constructed a correlation network of gut mucosal microbiomes from colorectal cancer patients $(n=86)$,

454 using 117 most representative ASVs, defined as ones with abundance of at least 10 sequences detected in 455 at least 15 samples. This filtering resulted in a median sample retainment rate of $77 \%$ [70\% - 85\%]. Zero

456 counts were imputed using the "CmultRepl" function, and the correlation network was constructed using

457 CCLasso, with 250 bootstrap and three-fold cross validation [27]. Interactions with adjusted p values <

$458 \quad 0.01$ and absolute correlation strength $>0.37$ were considered significant hits. Additionally, a separate

459 correlation network was inferred using SpiecEasi (Meinshausen-Buhlmann's neighbourhood selection,

460 nlambda=20) on the same dataset [28]. Both these methods have been demonstrated to produce robust

461 performance in a recent benchmark study [75]. To avoid spurious hits, only significant interactions

462 detected by both the CCLasso and SpiecEasi approaches were included in the final visualization. We

463 applied the same procedures to construct correlation networks of microbiomes in saliva samples $(\mathrm{n}=66$,

464115 ASVs) and controls' gut mucosa (n=43, 90 ASVs).

Fusobacterium isolation and whole genome sequencing

467 Fusobacterium isolation was performed on six selected case patients (P10, P16, P18, P28, P40, P46),

468 whose Fusobacterium relative abundance in the tumour microbiome exceeded $0.5 \%$ as inferred by

469 microbiome profiling. The respective samples (saliva, tumour, and non-tumour mucosa) were subjected to

470 anaerobic culturing in a Whitley A35 anaerobic workstation (Don Whitley Scientific, UK) supplied with

$4715 \% \mathrm{CO}_{2}, 10 \% \mathrm{H}_{2}$, and $85 \%$ nitrogen gas, following the Fusobacterium isolation procedures established

472 previously [76]. Briefly, mucosal tissues were thawed on ice, and $~ 100 \mathrm{mg}$ tissues were aseptically

473 excised and anaerobically homogenized in Diluent A to maximize bacterial recovery. The suspension

$474(100 \mu \mathrm{L})$ was then plated onto the selective media at two-fold and four-fold dilutions (EG agar

475 supplemented with L-cysteine $\mathrm{HCl}, 50 \mathrm{ml} /$ liter of defibrinated sheep blood, $7 \mathrm{mg} /$ liter of crystal violet, 5

$476 \mathrm{mg} /$ liter of vancomycin, $30 \mathrm{mg} /$ liter of neomycin, and $25 \mathrm{mg} /$ liter of nalidixic acid; Sigma-Aldrich,

477 Germany). Thawed saliva samples were plated directly on the selective media, either undiluted or at two- 
478 fold dilutions. Plates were incubated at $37^{\circ} \mathrm{C}$ for $48-72 \mathrm{~h}$, and colonies (up to 10 ) resembling that of

479 Fusobacterium were picked from each plate and sub-cultured on new EG media to confirm purity and

480 select for single colonies. The isolate's taxonomic identities were queried using MALDI-TOF, and those

481 characterized as Fusobacterium species were retained. A total of 56 Fusobacterium isolates were

482 recovered and subjected to DNA extraction using the Wizard genomic extraction kit (Promega, USA). For

483 each isolate, $1 \mathrm{ng}$ DNA was used to prepare the sequencing library using the Nextera XT library

484 preparation kit, following the manufacturer's instruction. Normalized libraries were pooled and

485 sequenced on an Illumina MiSeq platform to generate 250 bp paired-end reads.

Pangenome analysis, phylogenetic reconstruction and screening for virulence genes

488 FASTQC was used to check the sequencing quality of each read pair [77], and Trimmomatic v0.36 was

489 used to trim sequencing adapters and low-quality reads (paired end option, SLIDINGWINDOW:10:22,

490 HEADCROP:15, MINLEN:50) [78]. For each isolate, the trimmed read set was input into Unicycler

491 v0.4.9 to construct the de novo assembly, using default parameters, and contigs of size over 500bp were

492 retained for downstream analyses [79]. The assemblies were checked for traces of contamination using

493 Checkm, and three assemblies were shown contaminated and discarded [80]. The resulting assemblies

494 were of adequate quality, with median size of 2,125,169 bp [IQR: 2,067,843 - 2,168,429], median

495 number of contigs of 133 [IQR: 86 - 173] and the median N50 of 35,535 bp [IQR: 21,685 - 51,953].

496 Prokka v1.13 was used to annotate the assemblies, using the well-annotated $F$. nucleatum 23726

497 (accessed via FusoPortal) as reference [81]. To provide preliminary taxonomic classification up to the

498 subspecies level, FastANI was used to calculate the average nucleotide identity (ANI) between the

499 individual assembly and a set of Fusobacterium references, with an ANI value $\geq 95 \%$ denoting a shared

500 species/subspecies [82]. The pangenomes of $57 \mathrm{~F}$. nucleatum/hwasookii isolates (38 sequenced herein

501 plus 19 references) and $25 \mathrm{~F}$. periodonticum isolates (15 sequenced herein plus 10 references) were

502 constructed separately using panX, which clusters orthologous proteins based on individual gene tree

503 construction and adaptive postprocessing instead of relying on fixed nucleotide identity cutoff [83]. The 
504 respective core genome from each species complex was aligned, with invariant sites removed, producing

505 SNP alignments of 89,900 bp (F. nucleatum/hwasookii complex) and 106,738 bp (F. periodonticum

506 complex). These were input into RAxML to construct maximum likelihood phylogenies, under the

507 GTRGAMMA substitution model with 300 rapid bootstraps [84]. Using the pangenome analysis output

508 (clustered orthologous proteins and gene presence/absence matrix), we screened for the presence of

509 several known Fusobacterium virulence genes (fap2, fadA, $\operatorname{radD}, \operatorname{cbp} F$ ). The intact presence or synteny

510 of each genetic element was checked manually by gene alignment (Seaview) or genome visualization

511 (Artemis) tools [85,86]. Since Fap2 is a large protein $(3,000-4,600$ amino acid) with varying size among

512 the Fusobacterium species/subspecies, we checked for its intactness using ARIBA and mapping approach,

513 with clone-specific fap2 serving as mapping reference [87]. Visualization of phylogenetic tree and

514 associated metadata was performed using package 'ggtree' [88]. Individual protein sets were aligned and

515 inspected in Seaview, and phylogenies were constructed in RAxML, using the PROTGAMMAGTR

516 model and 200 rapid bootstraps.

\section{Intra-clonal variation examination}

519 To investigate intra-clonal variation with high confidence, we examined single nucleotide variants (SNV)

520 among isolates belonging to the same phylogenetic cluster (Figure 4 and Table 2), using the mapping

521 approach recommended previously [89]. For each phylogenetic cluster, trimmed fastq files from the

522 isolates were concatenated and input into Unicycler to construct a pan-assembly, with contigs less than

$523500 \mathrm{bp}$ removed. This pan-assembly was ordered against an appropriate Fusobacterium reference using

524 ABACAS, depending on its respective species/subspecies, creating a pseudogenome reference [90].

525 Trimmed paired-end reads from each isolate were mapped against this reference using a custom wrapper

526 script. Briefly, mapping was conducted using BWA MEM algorithm and samtools v1.8 [91,92], with

527 duplicate reads removed using PICARD, followed by indel realignment by GATK [93]. Reads with

528 nonoptimal local alignment were subsequently removed using samclip (--max 50;

529 github.com/tseemann/samclip), to avoid false positives during variant calling. SNVs were detected using 
530 the haplotype-based caller Freebayes [94], and low quality SNVs were removed using bcftools if they met

531 any of the following criteria: consensus quality $<30$, mapping quality $<30$, read depth $<4$, ratio of SNVs

532 to reads at a position $(\mathrm{AO} / \mathrm{DP})<85 \%$, coverage on the forward or reverse strand $<1$ [95]. Mapping

533 coverage at each position was inferred using samtools "depth" command (-a -Q 30). The bcftools

534 'consensus' command was used to generate a pseudosequence (with length identical to the mapping

535 reference), integrating the filtered SNVs and invariant sites, and masking the low mapping region (depth

536 4) and low-quality SNVs with 'N'. The presence of high quality SNVs were validated by manual

537 visualization of output bam files in Artemis, and SNV pertaining to recombination, transposons, plasmids,

538 or repetitive elements were excluded from interpretation.

\section{Data availability}

541 Raw sequence data are available in the NCBI Sequence Read Archive, including ones for 16S rRNA

542 sequencing (BioProject PRJNA791834) and Fusobacterium whole genome sequencing (BioProject

543 PRJNA791829). Source data and R codes used for the microbiome analysis and visualization are

544 deposited in Github (https://github.com/Hao-Chung/Vietnam_CRC_microbiome). 


\section{$546 \quad$ Figure legends}

547 Figure 1 The salivary and gut mucosa microbiomes of colorectal cancer patients. Principal coordinate

548 analyses (PCoA), conducted on phylogenetic-assisted isometric log-ratio (PhILR) transformed data, of (A)

54966 salivary microbiomes, and (B) 129 gut mucosa microbiomes, with different CRC groups and sample

550 types denoted by different colours (see Keys; biopsies and polyps collected from controls, nontumours

551 and tumours collected from cases). (C) Boxplot showing the distribution of pairwise beta-diversity,

552 calculated on PhILR transformed values, observed in each gut microbiome category. (D) Heatmap

553 displaying the proportional abundances of 24 most abundant genera, with headers showing the samples'

554 community state type (CST): CST1 (light gray), CST2 (dark gray), and the corresponding sample type:

555 biopsy (light blue), polyp (dark blue), nontumour (pink), tumour (dark red). Genera were coloured

556 according to their classifications at Phylum level (see Keys). Genera in black box represent ones with

557 probable origin from the oral cavity.

559 Figure 2 Bacterial taxa significantly abundant among the examined classes. Taxa, or amplicon sequence 560 variants (ASVs), were determined as significant and visualized in (A) and (B) if they were detected in at 561 least two of the three tested approaches (ANCOMBC, DESeq2, corncob; adjusted p-value $\leq 0.05$ ). (A)

562 Log2 fold change of ASVs that differ between paired tumour and non-tumour mucosal microbiomes from 563 case participants, using the full model 'Patient + sample type' (n=86). (B) Log2 fold change of ASVs that 564 differ between tumour and biopsy (control) mucosal microbiomes ( $n=67)$. Log2 fold change was derived 565 from ANCOMBC test output, and taxa of oral origin were coloured in pink. (C) Relative abundance of 566 ASVs assigned as Fusobacterium mortiferum (n=14), Fusobacterium nucleatum (n=14), Leptotrichia $567(\mathrm{n}=16)$, and Collinsella $(\mathrm{n}=14)$ in the tumour and nontumour mucosal microbiomes, stratified by cancer 568 stages (III-IV vs. II).

570 Figure 3 Correlation network of colorectal cancer gut mucosal microbiomes. The network was 571 constructed from 117 most representative ASVs sampled from 86 mucosal microbiomes, outlining 
572 significant interactions detected by both CCLasso ( $\mathrm{p}$ value $\leq 0.01$ and absolute correlation strength $>0.37$ )

573 and SpiecEasi. Positive and negative interactions were coloured as red and blue lines respectively, with

574 line weight proportional to correlation strength. The ASVs (nodes) were coloured based on taxonomic

575 family (see Legend), with sizes proportional to their relative abundances. The light green shaded area

576 entails ASVs identified as members of the human oral microbiome (comparison with expanded Human

577 Oral Microbiome Database); the blue shaded area covers ASVs identified as gut anaerobic commensals

578 (Lachnospirales, Bacteroidales, Bifidobacteriales, Oscillospirales); and gray shaded area covers other

579 tumour-associated ASVs (as identified in Figure 2A).

581 Figure 4 Maximum likelihood phylogenies of Fusobacterium isolates from this study. (A) F. nucleatum

582 species phylogeny constructed from the alignment of 516 core genes ( 89,900 variant sites; $\mathrm{N}=57)$, using $F$.

583 hwasookii clade as an outgroup. (B) F. periodonticum species phylogeny constructed from alignment of

584863 core genes $(106,738$ variant sites, $\mathrm{N}=26)$. Red circles at internal nodes denote bootstrap values $\geq 80$.

585 The associated metadata on the right describe the patient ID and clinical origin of isolates (where

586 reference genomes were left blank), and the genomic presence of several virulence factors (fap $2, f a d A$,

$587 f a d A 2, \operatorname{radD}, f a d A 3, c b p F)$. Light blue shaded area covers isolates identified as novel $F$. periodonticum

588 subspecies. The scale bars denote the estimated number of substitutions. 
Table 1 Baseline characteristics of patients recruited in this study. Overweight/obesity was classified using WHO recommendation for Asian populations. Oral diseases include self-reported gingivitis, periodontitis or halitosis. The number in each cell refers to median with interquartile range in brackets, percentage or count number for each category.

\begin{tabular}{|c|cc|c|}
\hline & CRC cases (n=42) & Controls (n=21) & p-value \\
\hline Age & $64[54-69]$ & $60[53-66]$ & 0.359 \\
Male sex & $62 \%$ & $76 \%$ & 0.395 \\
BMI & $22.9[20.85-24.95]$ & $22.2[21.1-23.4]$ & 0.387 \\
Overweight/obesity & $47.60 \%$ & $33 \%$ & 0.409 \\
Diabetes & $19 \%$ & $19 \%$ & 1 \\
High blood pressure & $52 \%$ & $47.60 \%$ & 0.79 \\
Active smoking in the last two years & $21.40 \%$ & $19 \%$ & 1 \\
Oral diseases & $33 \%$ & $38 \%$ & 0.782 \\
Family history of cancer & $19 \%$ & $19 \%$ & 1 \\
Location of sampled mucosa & & & 0.533 \\
Descending colon & 7 & 3 & \\
Sigmoid colon & 28 & 12 & \\
Rectum & 7 & $1[0.7-1.2]$ & \\
Size of tumour/polyp (cm) & $5[4-5.75]$ & & \\
TNM stage of cancer & II (18), III (20), IV (4) & low (4), none (17) & \\
Polyp dysplasia grade & &
\end{tabular}


Table 2 Summary of Fusobacterium isolates recovered from this study. Species names in italic represent Fusobacterium nucleatum subspecies, and $(*)$ denotes a potential new subspecies. Fusobacterium relative abundance, inferred from 16S rRNA gene profiling results, showed the values for non-tumour/tumour samples for each cancer patient. SNV: single nucleotide variant.

\begin{tabular}{|c|c|c|c|c|c|c|c|c|}
\hline $\begin{array}{c}\text { Phylogenetic } \\
\text { cluster }\end{array}$ & Species & Isolation source & $\begin{array}{c}\text { Number } \\
\text { of } \\
\text { isolates }\end{array}$ & $\begin{array}{c}\text { intraclonal } \\
\text { SNV }\end{array}$ & $\begin{array}{l}\text { Patient } \\
\text { ID }\end{array}$ & $\begin{array}{l}\text { Tumour } \\
\text { location }\end{array}$ & $\begin{array}{c}\text { Cancer } \\
\text { stage }\end{array}$ & $\begin{array}{c}\text { Fusobacterium } \\
\text { relative } \\
\text { abundance }\end{array}$ \\
\hline T10_Fa1 & animalis & tumour & 2 & 5 & $\mathrm{P} 10$ & Sigmoid & IIB & $0.026 / 0.24$ \\
\hline S16-12 & polymorphum & oral & 1 & & \multirow[b]{4}{*}{$\mathrm{P} 16$} & \multirow[b]{4}{*}{ Sigmoid } & \multirow[b]{4}{*}{ IIB } & \multirow[b]{4}{*}{$0.1 / 0.34$} \\
\hline S16-17 & periodonticum & oral & 1 & & & & & \\
\hline S16_Fa & animalis & oral & 2 & 10 & & & & \\
\hline TH16_Fvi & vincentii & tumour-nontumour & 6 & 1 & & & & \\
\hline H18-18 & animalis & nontumour & 1 & & \multirow[b]{5}{*}{$\mathrm{P} 18$} & \multirow[b]{5}{*}{ Descending } & \multirow[b]{5}{*}{ IIA } & \multirow[b]{5}{*}{$0.003 / 0.008$} \\
\hline S18-79 & polymorphum & oral & 1 & & & & & \\
\hline S18-78 & hwasookii & oral & 1 & & & & & \\
\hline S18_Fh & hwasookii & oral & 2 & 4 & & & & \\
\hline TH18_novelFperi & novel periodonticum* & tumour-nontumour & 6 & 2 & & & & \\
\hline S28-2 & periodonticum & oral & 1 & & \multirow[b]{2}{*}{$\mathrm{P} 28$} & \multirow[b]{2}{*}{ Sigmoid } & \multirow[b]{2}{*}{ IV } & \multirow[b]{2}{*}{$0.14 / 0.39$} \\
\hline T28_Fn & nucleatum & tumour & 3 & 1 & & & & \\
\hline S40-28 & animalis & oral & 1 & & \multirow[b]{3}{*}{$\mathrm{P} 40$} & \multirow[b]{3}{*}{ Sigmoid } & \multirow[b]{3}{*}{ IIIB } & \multirow[b]{3}{*}{$0.27 / 0.1$} \\
\hline TH40_novelFperi & novel periodonticum* & tumour-nontumour & 2 & 2 & & & & \\
\hline S40_Fp & polymorphum & oral & 2 & 2 & & & & \\
\hline S46-13 & vincentii & oral & 1 & & $\mathrm{P} 46$ & Sigmoid & IIIB & $0.038 / 0.2$ \\
\hline
\end{tabular}


600

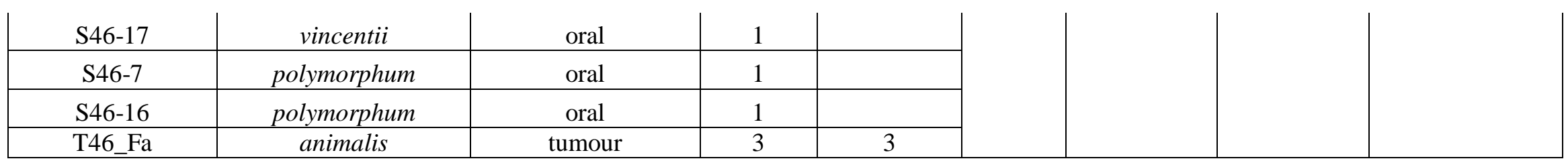

601

602 


\section{Funding information}

604 HCT is a Wellcome International Training Fellow (218726/Z/19/Z). LJH is supported by Wellcome Trust

605 Investigator Awards 100974/C/13/Z and 220876/Z/20/Z; the Biotechnology and Biological Sciences

606 Research Council (BBSRC), Institute Strategic Programme Gut Microbes and Health BB/R012490/1, and

607 its constituent projects BBS/E/F/000PR10353 and BBS/E/F/000PR10356. SB is a Wellcome Senior

608 Research Fellow (215515/Z/19/Z).

609

\section{Disclosure of potential conflicts of interest}

611 The authors report no potential conflicts of interest.

\section{2}

\section{Consent for publication}

614 This study does not publish any identifiable details related to the participant.

\section{Acknowledgements}

617 The authors wish to thank all participants and their caretakers for their participation in the study, and the 618 pathology laboratory of Binh Dan Hospital for the tumour/biopsy pathology results. 


\section{References}

1. Bray F, Ferlay J, Soerjomataram I, Siegel RL, Torre LA, Jemal A: Global cancer statistics 2018: GLOBOCAN estimates of incidence and mortality worldwide for 36 cancers in 185 countries. CA Cancer J Clin 2018, 68:394-424.

2. Arnold M, Sierra MS, Laversanne M, Soerjomataram I, Jemal A, Bray F: Global patterns and trends in colorectal cancer incidence and mortality. Gut 2017, 66:683-691.

3. Keum NN, Giovannucci E: Global burden of colorectal cancer: emerging trends, risk factors and prevention strategies. Nat Rev Gastroenterol Hepatol 2019, 16:713-732.

4. Schirmer M, Smeekens SP, Vlamakis H, Jaeger M, Oosting M, Franzosa EA, Jansen T, Jacobs L, Bonder MJ, Kurilshikov A, et al.: Linking the Human Gut Microbiome to Inflammatory Cytokine Production Capacity. Cell 2016, 167:1125-1136.e8.

5. Song M, Chan AT: Environmental Factors, Gut Microbiota, and Colorectal Cancer Prevention. Clin Gastroenterol Hepatol 2019, 17:275-289.

6. Castellarin M, Warren L, Freeman JD, Dreolini L, Krzywinski M, Strauss J, Barnes R, Watson P, Allen-vercoe E, Moore RA, et al.: Fusobacterium nucleatum infection is prevalent in human colorectal carcinoma. Genome Res 2012, 22:299-306.

7. Kostic AD, Gevers D, Pedamallu CS, Kostic AD, Gevers D, Pedamallu CS, Michaud M, Duke F, Earl AM, Ojesina AI, et al.: Genomic analysis identifies association of Fusobacterium with colorectal carcinoma. Genome Res 2012, 22:292-298.

8. Flemer B, Lynch DB, Brown JMR, Jeffery IB, Ryan FJ, Claesson MJ, O'Riordain M, Shanahan F, O'Toole PW: Tumour-associated and non-tumour-associated microbiota in colorectal cancer. Gut 2017, 66:633-643.

9. Wirbel J, Pyl PT, Kartal E, Zych K, Kashani A, Milanese A, Fleck JS, Voigt AY, Palleja A, Ponnudurai R, et al.: Meta-analysis of fecal metagenomes reveals global microbial signatures that are specific for colorectal cancer. Nat Med 2019, 25:679-689.

10. Thomas AM, Manghi P, Asnicar F, Pasolli E, Armanini F, Zolfo M, Beghini F, Manara S, Karcher 

microbial diagnostic signatures and a link with choline degradation. Nat Med 2019, 25:667678.

649 11. Brennan CA, Garrett WS: Fusobacterium nucleatum - symbiont, opportunist and oncobacterium. Nat Rev Microbiol 2018, doi:10.1038/s41579-018-0129-6.

651 12. Rubinstein MR, Wang X, Liu W, Hao Y, Cai G, Han YW: Fusobacterium nucleatum Promotes Colorectal Carcinogenesis by Modulating E-Cadherin/ $\beta$-Catenin Signaling via its FadA Adhesin. Cell Host Microbe 2013, 14:195-206.

654 13. Abed J, Emgård JEM, Zamir G, Faroja M, Almogy G, Grenov A, Sol A, Naor R, Pikarsky E, Atlan KA, et al.: Fap2 Mediates Fusobacterium nucleatum Colorectal Adenocarcinoma Enrichment by Binding to Tumor-Expressed Gal-GalNAc. Cell Host Microbe 2016, 20:215225.

14. Casasanta MA, Yoo CC, Udayasuryan B, Sanders BE, Umana A, Zhang Y, Peng H, Duncan AJ, Wang Y, Li L, et al.: Fusobacterium nucleatum host cell binding and invasion induces IL-8 and CXCL1 secretion that drives colorectal cancer cell migration. Sci Signal 2020, 1:1-13. 2020, 39:144-151.

16. Guo P, Tian Z, Kong X, Yang L, Shan X, Dong B, Ding X, Jing X, Jiang C, Jiang N, et al.: FadA promotes DNA damage and progression of Fusobacterium nucleatum-induced colorectal cancer through up-regulation of chk2. J Exp Clin Cancer Res 2020, 39:1-13.

17. Yu TC, Guo F, Yu Y, Sun T, Ma D, Han J, Qian Y, Kryczek I, Sun D, Nagarsheth N, et al.:

670 18. Mima K, Nishihara R, Qian ZR, Cao Y, Sukawa Y, Nowak JA, Yang J, Dou R, Masugi Y, Song 
673 19. Serna G, Ruiz-Pace F, Hernando J, Alonso L, Fasani R, Landolfi S, Comas R, Jimenez J, Elez E,

676 20. Salvucci M, Crawford N, Stott K, Bullman S, Longley DB, Prehn JHM: Patients with mesenchymal tumours and high Fusobacteriales prevalence have worse prognosis in

679 21. Bullman S, Pedamallu CS, Sicinska E, Clancy TE, Zhang X, Cai D, Neuberg D, Huang K, Guevara F, Nelson T, et al.: Analysis of Fusobacterium persistence and antibiotic response in colorectal cancer - supplementary. Science (80- ) 2017, 358:1443-1448. reveal worldwide activity inequality. Nature 2017, 547:336-339. 13. Commun 2020, 11:1-11.

25. Love MI, Huber W, Anders S: Moderated estimation of fold change and dispersion for RNAseq data with DESeq2. Genome Biol 2014, 15:550.

691 26. Martin BD, Witten D, Willis AD: Modeling microbial abundances and dysbiosis with betabinomial regression. Ann Appl Stat 2020, 14:94-115.

693 27. Fang H, Huang C, Zhao H, Deng M: CCLasso: Correlation inference for compositional data 694 through Lasso. Bioinformatics 2015, 31:3172-3180.

695 28. Kurtz ZD, Müller CL, Miraldi ER, Littman DR, Blaser MJ, Bonneau RA: Sparse and 696 Compositionally Robust Inference of Microbial Ecological Networks. PLOS Comput Biol 2015, 11:e1004226. 
698 29. Brewer ML, Dymock D, Brady RL, Singer BB, Virji M, Hill DJ: Fusobacterium spp. target human CEACAM1 via the trimeric autotransporter adhesin CbpF. J Oral Microbiol 2019, 11.

30. Wu C, Chen YW, Scheible M, Chang C, Wittchen M, Lee JH, Luong TT, Tiner BL, Tauch A, Das nucleatum. Proc Natl Acad Sci U S A 2021, 118.

703 31. Galaski J, Shhadeh A, Umaña A, Yoo CC, Arpinati L, Isaacson B, Berhani O, Singer BB, Slade

712 34. Meng Q, Gao Q, Mehrazarin S, Tangwanichgapong K, Wang Y, Huang Y, Pan Y, Robinson S, pathogenicity. EMBO Rep 2021, 22:1-19.

35. Gao Z, Guo B, Gao R, Zhu Q, Qin H: Microbiota disbiosis is associated with colorectal cancer.

717 36. Torres PJ, Fletcher EM, Gibbons SM, Bouvet M, Doran KS, Kelley ST: Characterization of the salivary microbiome in patients with pancreatic cancer. PeerJ 2015, 2015:1-16. 
39. Liang JQ, Li T, Nakatsu G, Chen YX, Yau TO, Chu E, Wong S, Szeto CH, Ng SC, Chan FKL, et adenoma and cancer. Gut 2019, 69:1248-1257.

40. Chung The H, Sessions PF de, Jie S, Thanh DP, Thompson CN, Minh CNN, Chu CW, Tran T-A, Thomson NR, Thwaites GE, et al.: Assessing gut microbiota perturbations during the early phase of infectious diarrhea in Vietnamese children. Gut Microbes 2018, 9:38-54.

41. Liang S, Mao Y, Liao M, Xu Y, Chen Y, Huang X, Wei C, Wu C, Wang Q, Pan X, et al.: Gut microbiome associated with APC gene mutation in patients with intestinal adenomatous

42. Wei PL, Hung CS, Kao YW, Lin YC, Lee CY, Chang TH, Shia BC, Lin JC: Classification of changes in the fecal microbiota associated with colonic adenomatous polyps using a long-

43. He Y, Mujagond P, Tang W, Wu W, Zheng H, Chen X, Chen M, Ma W, Chen G, Zhou H: Noncolorectal cancer-associated FadA virulence factor. Gut 2020, 69:1998-2007.

45. Robrish SA, Oliver C, Thompson J: Sugar metabolism by fusobacteria: regulation of transport, Immun 1991, 59:4547-54.

46. Lagier JC, Dubourg G, Million M, Cadoret F, Bilen M, Fenollar F, Levasseur A, Rolain JM, 
Population. Microbiol Spectr 2021, doi:10.1128/spectrum.00526-21.

753 48. Richardson M, Ren J, Rubinstein MR, Taylor JA, Friedman RA, Shen B, Han YW: Analysis of 16S rRNA genes reveals reduced Fusobacterial community diversity when translocating from saliva to GI sites. Gut Microbes 2020, 12:1-13.

756 49. Abed J, Maalouf N, Manson AL, Earl AM, Parhi L, Emgård JEM, Klutstein M, Tayeb S, Almogy G, Atlan KA, et al.: Colon Cancer-Associated Fusobacterium nucleatum May Originate From the Oral Cavity and Reach Colon Tumors via the Circulatory System. Front Cell Infect

50. Ailloud F, Didelot X, Woltemate S, Pfaffinger G, Overmann J, Bader RC, Schulz C, Malfertheiner P, Suerbaum S: Within-host evolution of Helicobacter pylori shaped by niche-specific adaptation, intragastric migrations and selective sweeps. Nat Commun 2019, 10:2273. Both? Trends in Cancer 2021, 7:185-187.

768 53. WHO expert consultation: Appropriate body-mass index for Asian populations and its implications for policy and intervention strategies. Lancet 2004, 363:157-63.

770 54. American Joint Committee on Cancer: Chapter 20 - Colon and Rectum. Springer; 2017.

771 55. Kozich JJ, Westcott SL, Baxter NT, Highlander SK, Schloss PD: Development of a dual-index sequencing strategy and curation pipeline for analyzing amplicon sequence data on the

774 56. Gohl D, Gohl DM, MacLean A, Hauge A, Becker A, Walek D, Beckman KB: An optimized 775 protocol for high-throughput amplicon-based microbiome profiling. Protoc Exch 2016, 
doi:10.1038/protex.2016.030.

777 57. Eisenhofer R, Minich JJ, Marotz C, Cooper A, Knight R, Weyrich LS: Contamination in Low 2019, 27:105-117.

58. R Core Team: R: A language and environment for statistical computing. 2016,

59. McMurdie PJ, Holmes S: phyloseq: an R package for reproducible interactive analysis and graphics of microbiome census data. PLoS One 2013, 8:e61217.

60. Silverman JD, Washburne AD, Mukherjee S, David LA: A phylogenetic transform enhances analysis of compositional microbiota data. Elife 2017, 6:1-20.

61. Oksanen J, Blanchet G, Kindt R, Legendre P, Minchin P, O’Hara R, Simpson G, Solymos P, Stevens M, Wagner H: vegan: Community Ecology Package. R package version 2.3-5. 2016,

62. Callahan BJ, Sankaran K, Fukuyama JA, McMurdie PJ, Holmes SP: Bioconductor workflow for

63. Callahan BJ, McMurdie PJ, Holmes SP: Exact sequence variants should replace operational taxonomic units in marker-gene data analysis. ISME J 2017, doi:10.1038/ismej.2017.119.

792 64. Wang Q, Garrity GM, Tiedje JM, Cole JR: Naive Bayesian classifier for rapid assignment of rRNA sequences into the new bacterial taxonomy. Appl Environ Microbiol 2007, 73:5261-7.

794 65. Mirarab S, Nguyen N, Guo S, Wang L-S, Kim J, Warnow T: PASTA: Ultra-Large Multiple 
(compositional) data. Sci Total Environ 2010, 408:4230-4238.

803 69. Tibshirani R, Walther G, Hastie T: Estimating the number of clusters in a data set via the gap statistic. J R Stat Soc 2001, 63:411-423.

805 70. Ishwaran H, Kogalur UB: Random Forests for Survival, Regression and Classification (RF-

$806 \quad$ SRC), R package version 2.2.0. 2016,

807 71. Calgaro M, Romualdi C, Waldron L, Risso D, Vitulo N: Assessment of statistical methods from single cell, bulk RNA-seq, and metagenomics applied to microbiome data. Genome Biol 2020, 21:1-31.

72. Nearing JT, Douglas GM, Hayes M, Macdonald J, Desai D, Allward N, Jones CMA, Wright R, disturbingly different results across 38 datasets. bioRxiv 2021,

73. Stevens JR, Herrick JS, Wolff RK, Slattery ML: Power in pairs: Assessing the statistical value extraction from single-cell RNA-seq data. Nat Commun 2018, 9.

817 75. Hirano H, Takemoto K: Difficulty in inferring microbial community structure based on cooccurrence network approaches. BMC Bioinformatics 2019, 20:1-14.

819 76. Komiya Y, Shimomura Y, Higurashi T, Sugi Y, Arimoto J, Umezawa S, Uchiyama S, Matsumoto M, Nakajima A: Patients with colorectal cancer have identical strains of Fusobacterium nucleatum in their colorectal cancer and oral cavity. Gut 2018, 0:7-9.

822 77. Andrews S: FastQC: A Quality Control Tool for High Throughput Sequence Data. [date 823 unknown],

824 78. Bolger AM, Lohse M, Usadel B: Trimmomatic: A flexible trimmer for Illumina sequence data. 825 Bioinformatics 2014, 30:2114-2120.

826 79. Wick RR, Judd LM, Gorrie CL, Holt KE: Unicycler: Resolving bacterial genome assemblies from short and long sequencing reads. PLoS Comput Biol 2017, 13:1-22. 
828 80. Parks DH, Imelfort M, Skennerton CT, Hugenholtz P, Tyson GW: CheckM: Assessing the

831 81. Todd SM, Settlage RE, Lahmers KK, Slade DJ: Fusobacterium Genomics Using MinION and

82. Jain C, Rodriguez-R LM, Phillippy AM, Konstantinidis KT, Aluru S: High throughput ANI analysis of 90K prokaryotic genomes reveals clear species boundaries. Nat Commun 2018,

83. Ding W, Baumdicker F, Neher RA: panX $\square$ : pan-genome analysis and exploration. Nucleic Acids Res 2018, 46:1-12.

84. Stamatakis A: RAxML version 8: a tool for phylogenetic analysis and post-analysis of large phylogenies. Bioinformatics 2014, 30:1312-3.

85. Gouy M, Guindon S, Gascuel O: SeaView version 4: A multiplatform graphical user interface database. Bioinformatics 2008, 24:2672-2676.

88. Yu G, Smith DK, Zhu H, Guan Y, Lam TTY: ggtree: An r package for visualization and annotation of phylogenetic trees with their covariates and other associated data. Methods Ecol Evol 2016, 8:28-36. atopic dermatitis increases disease severity. bioRxiv 2021, 
854 90. Assefa S, Keane TM, Otto TD, Newbold C, Berriman M: ABACAS: Algorithm-based

855 automatic contiguation of assembled sequences. Bioinformatics 2009, 25:1968-1969.

856 91. Li H: Aligning sequence reads, clone sequences and assembly contigs with BWA-MEM.

857 bioarxiv 2013,

858 92. Li H, Handsaker B, Wysoker A, Fennell T, Ruan J, Homer N, Marth G, Abecasis G, Durbin R:

859 The Sequence Alignment/Map format and SAMtools. Bioinformatics 2009, 25:2078-9.

860 93. McKenna A, Hanna M, Banks E, Sivachenko A, Cibulskis K, Kernytsky A, Garimella K,

861 Altshuler D, Gabriel S, Daly M, et al.: The Genome Analysis Toolkit: A MapReduce

862 framework for analyzing next-generation DNA sequencing data. Genome Res 2010, 20:1297-

$863 \quad 303$.

864 94. Garrison E, Marth G: Haplotype-based variant detection from short-read sequencing. arXiv

$865 \quad$ Prepr 2012,

866 95. Danecek P, Bonfield JK, Liddle J, Marshall J, Ohan V, Pollard MO, Whitwham A, Keane T,

867 McCarthy SA, Davies RM, et al.: Twelve years of SAMtools and BCFtools. Gigascience 2021,

$868 \quad 10: 1-4$. 


\section{A}

Parabacteroides merdae Dorea formicigenerans Collinsella aerofaciens

Bacteroides vulgatus

Bacteroides nordii

Osillibacter ruminantium Streptococcus sanguinis Fusobacterium.

Lachnoclostridium Fusobacterium nucleatum. ণ্শে

뜨

Selenomonas sputigena

Campylobacter rectus

Hungatella

B

Fusobacterium mortiferum Tyzzerella Sutterella

Campylobacter rectus Selenomonas sputigena Leptotrichia Fusobacterium nucleatum . Peptostreptococcus Gemella $-5.0$ Bacteria of oral origin
Nontumour mucosa (CRC)

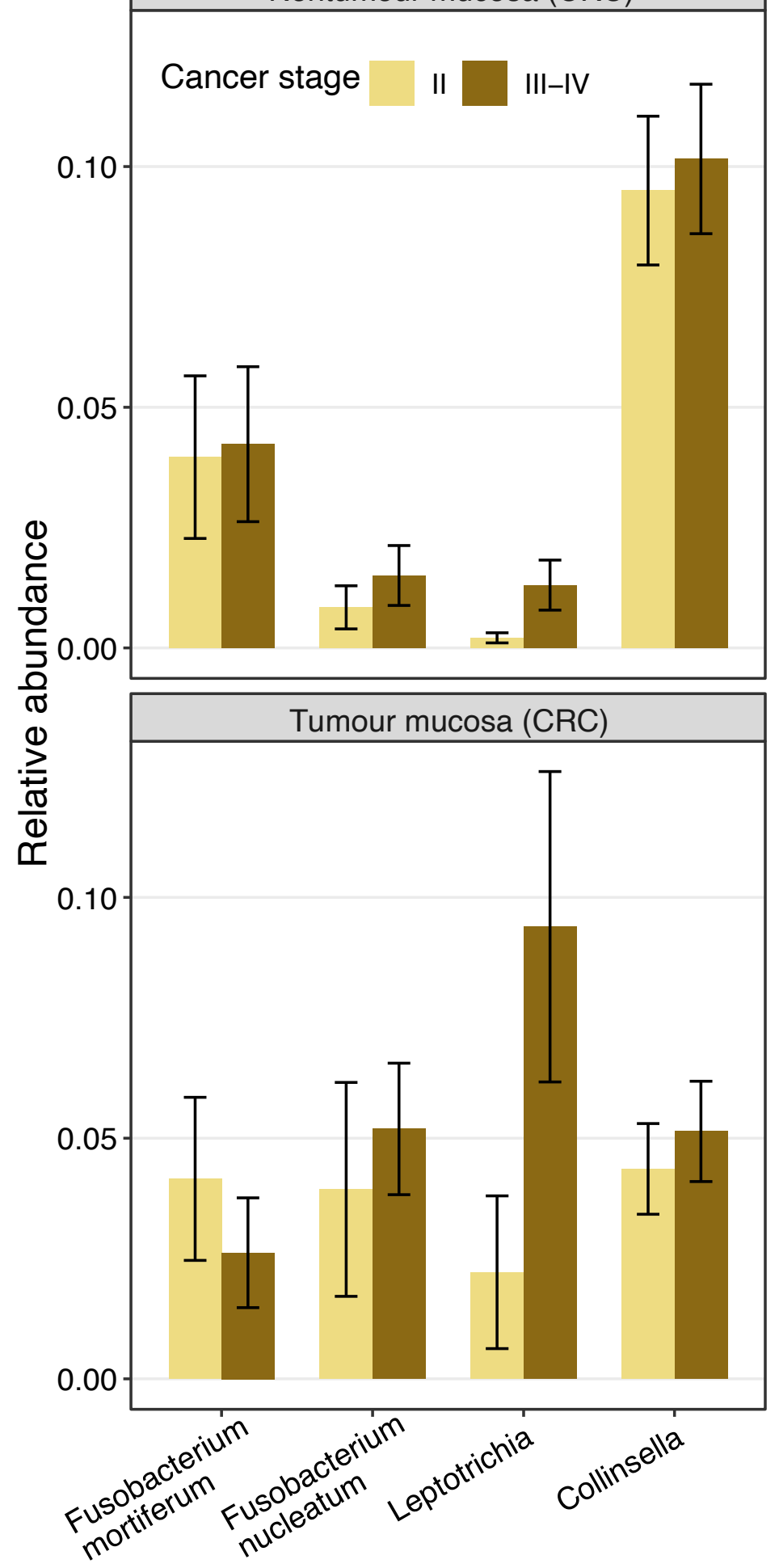




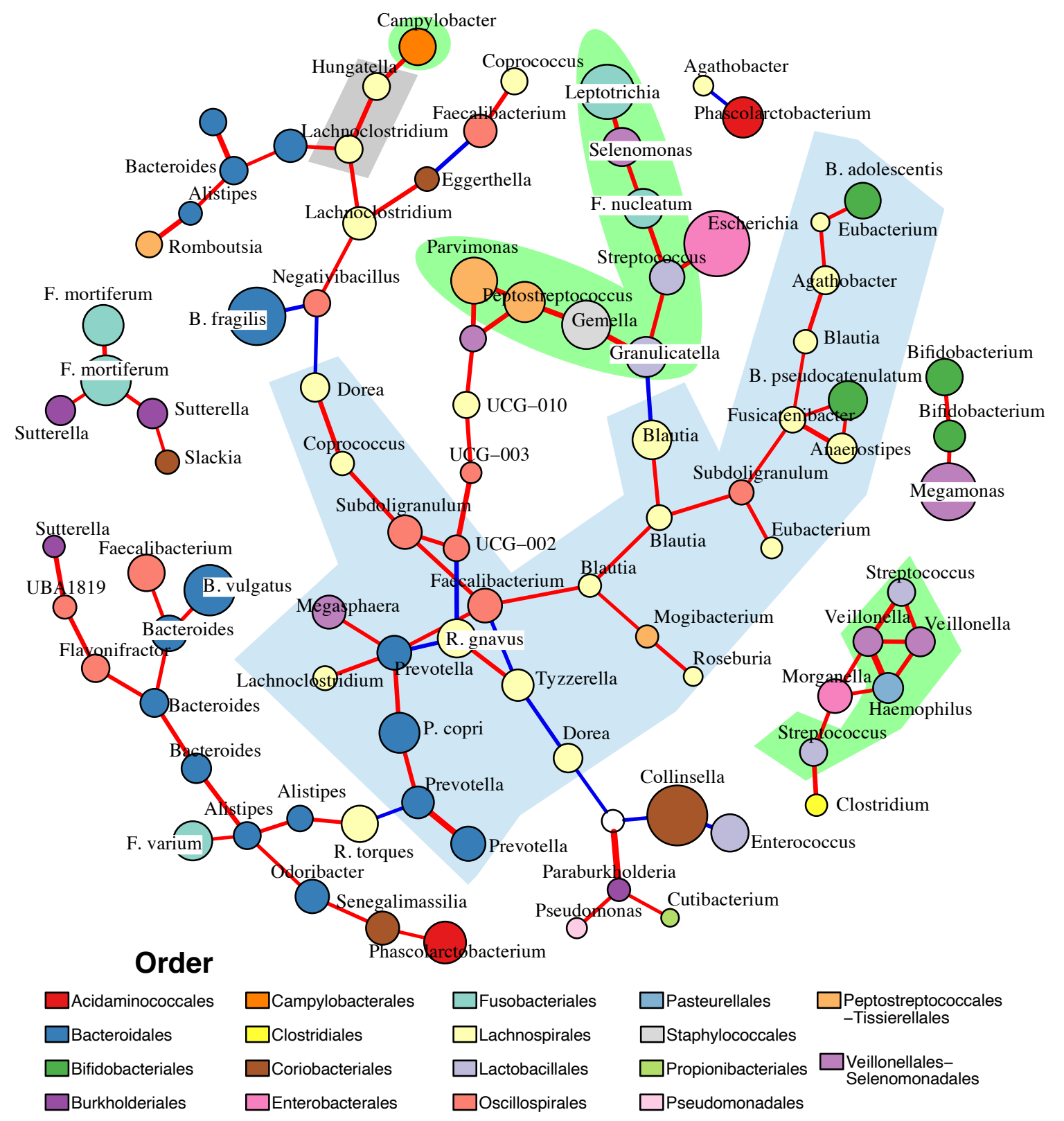




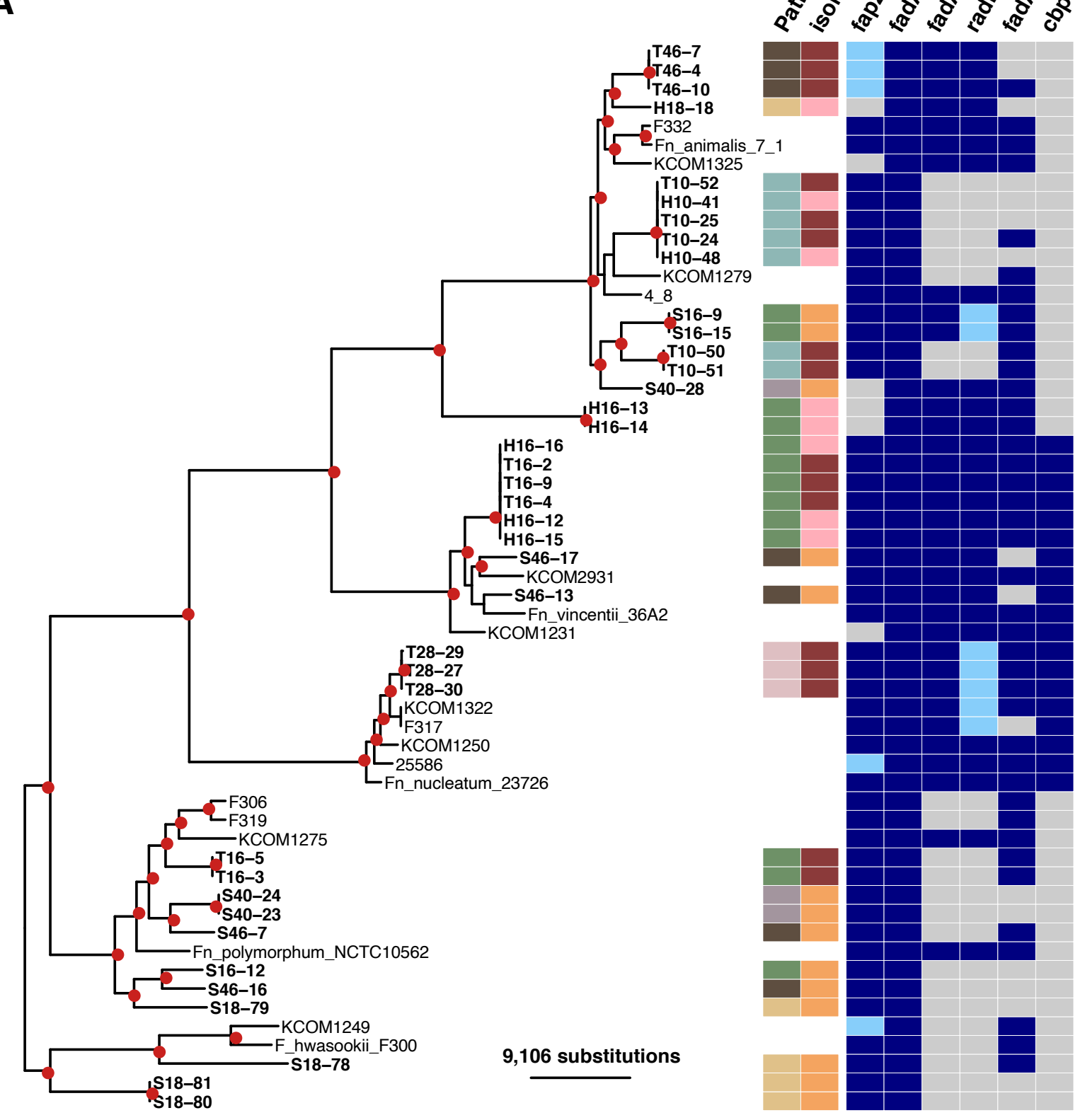

\begin{tabular}{|c|c|c|}
\hline \multicolumn{2}{|c|}{ Patient_ID } & \\
\hline 10 & 18 & 40 \\
16 & 28 & 46 \\
\hline
\end{tabular}

Isolation

oral

Gut (non-tumour)

\section{Virulence factors}

present disrupted absent

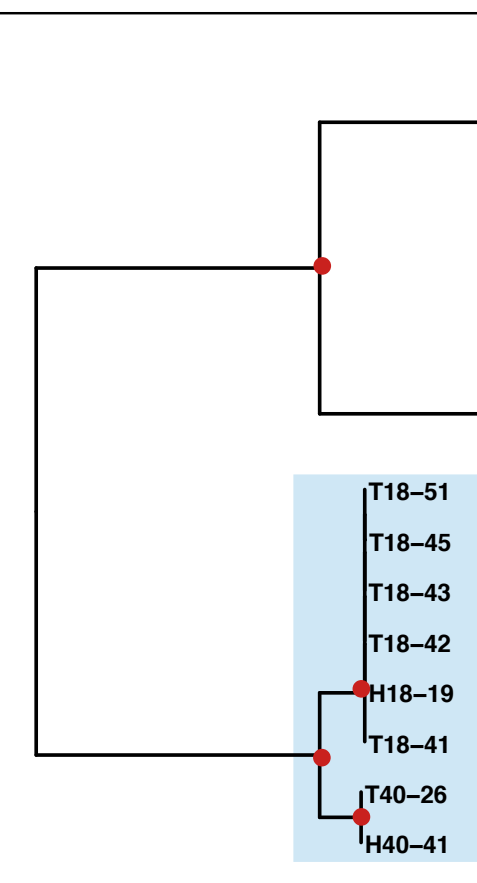

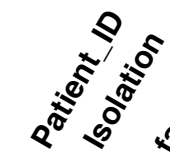

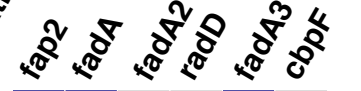

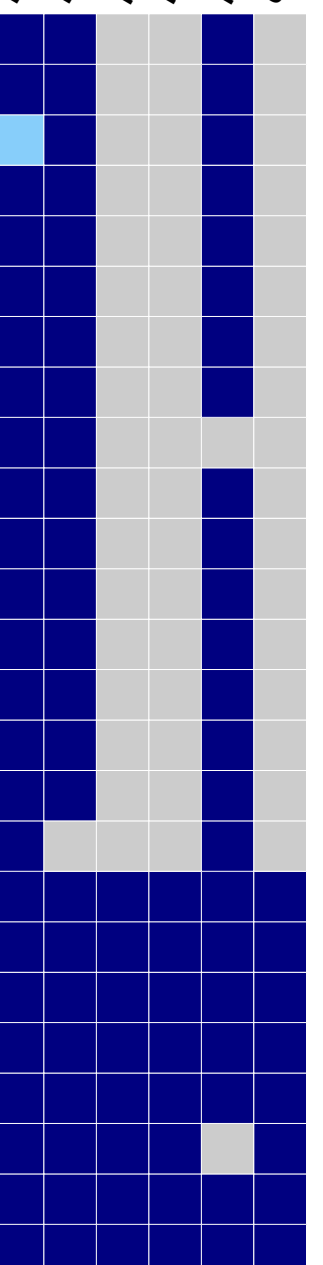

